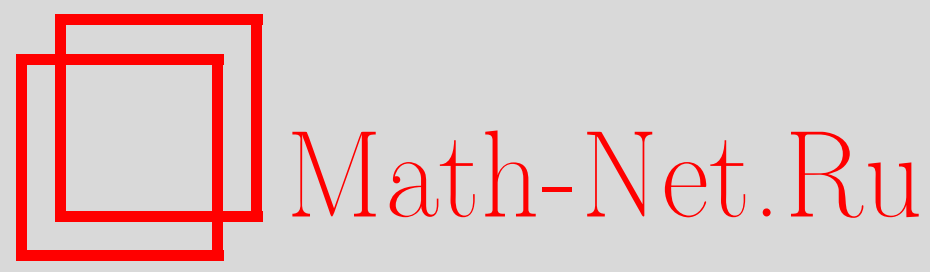

А. О. Иванов, А. А. Тужилин, Ле Хонг Ван, Нетривиальные критические сети. Особенности лагранжианов и критерий критичности, Матем. заметки, 2001, том 69, выпуск 4, 566-580

DOI: https://doi.org/10.4213/mzm523

Использование Общероссийского математического портала Math-Net.Ru подразумевает, что вы прочитали и согласны с пользовательским соглашением http://www.mathnet.ru/rus/agreement

Параметры загрузки:

IP : 3.80 .181 .102

26 апреля 2023 г., 16:06:31

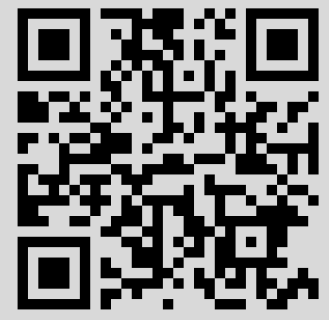




\title{
НЕТРИВИАЛЬНЫЕ КРИТИЧЕСКИЕ СЕТИ. ОСОБЕННОСТИ ЛАГРАНЖИАНОВ \\ И КРИТЕРИЙ КРИТИЧНОСТИ
}

\author{
А. О. Иванов, Хонг Ван Ле, А.А. Тужилин
}

\begin{abstract}
Выделен класс так называемых квазирегулярных лагранжианов, содержащих особенности на нулевом сечении кокасательной расслоения к многообразию, на котором рассматриваются экстремальные сети. Для таких лагранжианов доказан критерий экстремальности сети: на каждом ее ребре должны выполняться уравнения ЭйлераЛагранжа, а в вершинах - некоторые условия согласования.

Библиография: 4 названия.
\end{abstract}

Введение. В настоящей работе изучаются разветвленные экстремали (экстремальные сети) одномерных вариационных функционалов типа Лагранжа. При этом экстремальность понимается в следуюшем широком смысле: при деформации разрешается расщеплять вершины, меняя при этом топологию сети. Чтобы различать сети, для которых вершины расщеплять нельзя, и сети, допускающие расщепления, авторы назьвают первые - параметрическими сетями, т.е. сетями с фиксированной параметризацией, а вторые - сетями-следами, или просто сетями.

В центре внимания авторов следующие два вопроса:

- какие свойства лагранжианов приводят к существованию нетривиальных разветвлений его экстремальных сетей;

- какова локальная структура разветвленных экстремалей.

Отметим, что, вообще говоря, дополнительная свобода, возникающая из-за расщепления вершин, может существенно ограничить класс экстремалей по отношению к экстремальным параметрическим сетям и вообще привести к отсутствию экстремалей. Оказывается, наличие нетривиальных экстремалей тесно связано с наличием особенностей у лагранжиана, см. теорему 3.1 и следствия 3.1-3.4. Тривиальность экстремалей здесь понимается в следующем смысле: сеть называется тривиально экстремальной, если, во-первых, каждое ее ребро - экстремаль по отношению к произвольным деформациям (в частности, смещающим любые вершины сети) и, во-вторых, каждая точечная кривая, образ которой совпадает с произвольной вершиной сети, также является

Данная работа была выполнена авторами в Институте прикладной математики Макса Планка (г. Лейпциг, Германия), где А. О. Иванов и А. А. Тужилин работали по приглашению профессора Ю. Йоста в апреле-июне 1999 года. Работа первого и третьего авторов выполнена при частичной поддержке Российского фонда фундаментальных исследований, гранты № 96-15-96142 и № 98-01-00240, и фонда INTAS, грант № 97-0808. 
экстремалью по отношению к любьм деформациям. В частности, если разрезать тривиально экстремальную сеть по любому набору ее вершин, то в результате мы получим объединение сетей, вновь являюшихся экстремальными. Авторами показано, что если лагранжиан является гладким, то соответствующий функционал не имеет нетривиальных экстремалей.

Авторами введен в рассмотрение класс квазирегулярных лагранжианов, т.е. лагранжианов, имеющих, в некотором смысле, простейшие особенности, приводящие к появлению нетривиальных разветвленных экстремалей. Для случая таких лагранжианов авторами получен критерий экстремальности сети, см. теорему 3.2. Основная трудность при доказательстве этой теоремы состоит в необходимости следить за всевозможными расщеплениями вершин сети, количество же таких расщеплений, вообще говоря, неограничено.

Кроме того, получен критерий экстремальности квазирегулярной параметрической сети (теорема 2.1), а также критерий экстремальности следа в предположении гладкости квазирегулярного лагранжиана (теорема 3.1).

Авторы пользуются случаем выразить свою глубокую признательность профессору Ю. Йосту за проявленньй интерес к работе и предоставленную возможность плодотворно совместно поработать в MPI für Mathematik in den Naturwissenschaften, Leipzig. Авторы благодарны академику А. Т. Фоменко за постоянное внимание к работе.

1. Предварительные сведения. Мы будем рассматривать графы с топологической точки зрения. Топологическим графом $G$ назьвается топологическое пространство, полученное из конечной совокупности отрезков $\left\{I_{\alpha}\right\}$ некоторой склейкой по их концам. Пусть $\pi: \bigsqcup_{\alpha} I_{\alpha} \rightarrow G$ - каноническая проекция. Образы внутренностей отрезков $I_{\alpha}$ при отображении $\pi$ называются ребрами графа $G$, а $\pi$-образы концевых точек отрезков $I_{\alpha}-$ вершинами. Если для каждого $\alpha$ фиксирован конкретньй отрезок $\left[a_{\alpha}, b_{\alpha}\right]=I_{\alpha}$ числовой прямой, то такой граф называется оснащенным. Отметим, что так как конщы произвольного отрезка $\left[a_{\alpha}, b_{\alpha}\right]$ естественным образом упорядочены, а именно, $a_{\alpha}<b_{\alpha}$, то тем самым фиксирована ориентация каждого ребра оснащенного графа и можно говорить о его начальной и конечной вершинах.

Гомеоморфизм $\varphi: G_{1} \rightarrow G_{2}$ оснащенных графов назьвается әквивалентностью, если он переводит вершины в вершины и для каждого ребра $[a, b]$ графа $\left.G_{1} \varphi\right|_{[a, b]}$ есть тождественное отображение отрезка $[a, b]$ на себя. Оснащенные графы $G_{1}$ и $G_{2}$ назьваются әквивалентными, если существует эквивалентность $\varphi: G_{1} \rightarrow G_{2}$.

Предположим, что в графе $G$ выделено некоторое подмножество $B$ множества его вершин. Такой граф $G$ мы будем назьвать графом $c$ граниией $\partial G=B$. Вершины из $\partial G$ будем называть граничными или неподвижныьми, а все остальные вершины внутренними или подвижными. Ребра графа, инцидентные граничньм вершинам, также назовем граничными, а ребро, не инцидентное никакой граничной вершине, назовем внутренним.

ОПРЕДЕЛЕниЕ. Пусть $G$ - произвольньй связный оснащенный граф и $\partial G-$ его гранища. Параметрической (оснащенной) сетью топологии $G$ на многообразии $W$ назьвается непрерьвное отображение $\Gamma$ из $G$ в $W$. Граф $G$ в этом случае назьвается $n a-$ раметризующим графом параметрической сети Г или ее топологией.

Вся терминология теории графов, топологических пространств и их отображений непосредственно переносится на параметрические сети. Например, ограничение отображения Г на вершины, ребра, границу, связный подграф параметризующего графа, 
локальный графф и т.д. назьваются вершинами, ребрами, граниией, подсетью, и т.д. параметрической сети $Г$.

ЗАмЕчАниЕ. Вьше мы представили каждьй оснащенньй граф как набор отрезков, факторизованный по некоторой эквивалентности, отождествляющей концевые точки этих отрезков. Точно так же, параметрическую сеть можно представить как набор непрерьвных кривых в многообразии, некоторые концевые точки которых отождествлены.

Параметрическая сеть $\Gamma: G \rightarrow W$ называется гладкой (регулярной, кусочно-гладкой, кусочно-регулярной), если ограничение отображения Г на замыкание каждого ребра графа $G$ является таковым. Отметим, что понятие гладкой параметрической сети естественным образом обобщает понятие гладкой кривой.

Пусть $\Gamma: G \rightarrow X-$ произвольная параметрическая сеть и $I=[a, b]-$ некоторьй отрезок.

ОПреДЕЛЕНИЕ. Непрерьвное отображение $\Psi: G \times I \rightarrow W$ такое, что для всех $g \in G$ имеет место равенство $\Psi(g, a)=\Gamma(g)$, назьвается деформацией параметрической се$m u$ Г. Если исходная параметрическая сеть $Г$ является гладкой (регулярной, кусочно-гладкой, кусочно-регулярной), то мы будем предполагать, что каждая параметрическая сеть $\Psi(\cdot, t)=\Gamma_{t}$ является таковой и что для каждого замыкания $\bar{e}$ ребра е графа $G$ ограничение отображения $\Psi$ на $\bar{e} \times I$ является гладким или кусочно-гладким.

Пусть $\Gamma: G \rightarrow W$ - гладкая сеть на гладком многообразии $W$ и $\Gamma_{t}, \Gamma_{t=0}=\Gamma,-$ некоторая ее деформация. Рассмотрим произвольную точку $g \in G$, и пусть $\Gamma_{t}(g)$ - кривая, по которой движется точка $\Gamma(g)$ при деформации. Семейство векторов скоростей кривых $\Gamma_{t}(g)$ при $t=0$ по всем точкам $g \in G$ называется полем деформации $\Gamma_{t}$.

Введем на классе всех параметрических сетей на $W$ отношение эквивалентности $\rho$ следуюшим образом. Будем говорить, что параметрическая сеть $\Gamma_{1}$ может быть спроецирована на $\Gamma_{2}$, если существует проекция $\pi: G_{1} \rightarrow G_{2}$ такая, что $\Gamma_{2} \circ \pi=\Gamma_{1}$. Здесь под проекцией $\pi: G_{1} \rightarrow G_{2}$ мы понимаем каноническую проекцию пространства $G_{1}$ на фактор-пространство $G_{2}=G_{1} / H$, где $H$ - некоторьй связньй подграф в $G_{1}$. Проекция $\pi$ индуцирует отображение $\pi: \Gamma_{1} \rightarrow \Gamma_{2}$ одной сети в другую ${ }^{1}$, которое мы также будем назьвать проекиией. Две параметрических сети $\Gamma_{i}: G_{i} \rightarrow X, i=1,2$, назовем $\rho$-смежнылми, если одна из них может быть спроецирована на другую. Отметим, что отношение $\rho$-смежности рефлексивно и симметрично, но не транзитивно. Расширим его до отношения эквивалентности следующим образом. Две параметрических сети $\Gamma$ и $\Gamma^{\prime}$ назовем $\rho$-әквивалентными, если существует конечная последовательность $\left\{\Gamma=\Gamma_{1}, \Gamma_{2}, \ldots, \Gamma_{n}=\Gamma^{\prime}\right\}$ параметрических сетей такая, что каждые две соседних сети $\Gamma_{i}$ и $\Gamma_{i+1}$ в этой последовательности $\rho$-смежны. Классы $\rho$-эквивалентности называются (оснащенными) сетями-следами или просто (оснащенными) сетями. Если параметрическая сеть Г содержится в следе $\Upsilon$, то будем писать $\Upsilon=[\Gamma]$.

Каноническим представителем следа $\Upsilon$ называется такая параметрическая сеть $\Gamma \in \Upsilon$, что каждая параметрическая сеть $\Gamma^{\prime}$ из $\Upsilon$ может быть спроецирована на $\Gamma$. Можно показать, что у каждого следа существует единственньй с точностью до эквивалентности канонический представитель (см. [1]).

\footnotetext{
${ }^{1}$ Говоря про отображение одной сети в другую, мы имеем в виду отображение граффиков, соответствующих этим сетям. Напомним, что сети - это по определению отображения, а грабики - это множества, состоящие из пар.
} 
(Локальной) деформацией следа $\Upsilon$ назовем однопараметрическое семейство сетей $\Upsilon_{t}=\left[\Gamma_{t}\right], t \in\left[t_{1}, t_{2}\right]$, где $\Gamma_{t}$ - деформация некоторой параметрической сети $\Gamma=\Gamma_{t_{1}}$ из $\Upsilon$ такая, что все параметрические сети $\Gamma_{t}$ при $t>t_{1}$ являются каноническими представителями сетей $\Upsilon_{t}$.

2. Локальная структура экстремальных параметрических сетей. В этом пункте мы для простоты ограничиваемся рассмотрением лишш автономных лагранжианов, хотя все те же результаты могут быть получены и в неавтономном случае.

Пусть $W$ - гладкое многообразие, $\pi: T W \rightarrow W$-касательное расслоение, $L: T W \rightarrow$ $\mathbb{R}$ - непрерывный лагранжиан. Пусть $\Omega$ - пространство всех кусочно-гладких кривых на $W$ и $\psi_{L}: \Omega \rightarrow \mathbb{R}$ - классический вариационньй функционал, соответствующий лагранжиану $L$, т.е.

$$
\psi_{L}(\gamma)=\int_{a}^{b} L(\gamma(t), \dot{\gamma}(t)) d t
$$

где $\gamma:[a, b] \rightarrow W-$ кривая из $\Omega$.

Пусть $G$ - оснашенный граф, $\partial G$ - его граница, $\beta: \partial G \rightarrow W$ - произвольное отображение, $\Gamma: G \rightarrow W$ - произвольная кусочно-гладкая параметрическая сеть с границей $\partial \Gamma=\beta$. На множестве таких сетей определен функционал $\Psi_{L}$, значение которого на сети $\Gamma$ равно сумме значений функционала $\psi_{L}$ на ребрах этой сети.

Пусть $(x, \xi)$ - произвольная точка из $T W$. Предположим, что лагранжиан $L$ дважды непрерьвно дифференцируем в точке $(x, \xi)$. Обозначим через $p(x, \xi)=L_{\xi}(x, \xi)$ точку из кокасательного пространства $T_{x}^{*} W$, определенную следующим образом. Если $x^{i}-$ координаты на $W$ в окрестности точки $x$, а $\xi^{i}$ - соответствующие координаты в касательном пространстве $T_{x} W$, то $i$-я координата ковектора $p(x, \xi)$ имеет вид

$$
p(x, \xi)_{i}=\frac{\partial L}{\xi^{i}}(x, \xi) .
$$

Ковектор $p(x, \xi)$ называется (обобщенным) импульсом в точке $(x, \xi)$. Далее, определим другой ковектор $[L](x, \xi)$ следующим образом. Рассмотрим произвольную гладкую кривую $x(t)$, для которой $\dot{x}(0)=\xi$. Положим

$$
[L](x, \xi)_{i}=\left.\frac{d}{d t}\right|_{t=0}\left\{p_{i}(x(t), \dot{x}(t))\right\}-\frac{\partial L}{\partial x^{i}}(x(0), \dot{x}(0)) .
$$

Хорошо известно, что это определение не зависит от выбора кривой $x(t)$, см. [2]. Ковектор $[L]$ назьвается производной Лагранжа функиии $L$.

Кривую $\gamma(t)$ назовем квазирегулярной, если она либо регулярна, либо является отображением в точку. Параметрическую сеть Г назовем квазирегулярной, если все ее ребра - квазирегулярные кривые. Ребро графа $G$, параметризующее регулярное (точечное) ребро сети $\Gamma$, также будем называть регулярным (соответственно точечным).

Лагранжиан $L$ назовем квазирегулярным, если вьполнены следующие условия:

1 ) функция $L$ является гладкой на всем $T W$, кроме, быть может, нулевого сечения $W_{0} \subset T W$

2) ограничение функции $L$ на нулевое сечение $W_{0} \subset T W$ равно нулю;

$3)$ для любого вектора $\zeta \in T_{(P, \xi)}(T W)$ существует производная $\zeta(L)$ функции $L$ по направлению ${ }^{2}$ вектора $\zeta$;

\footnotetext{
${ }^{2}$ Здесь и всюду ниже производная функции по направлению понимается как односторонний предел. В частности, производные функции по противоположным направлениям, вообще говоря, не обязаны быть противоположны.
} 
4) для произвольной гладкой деформации $\gamma_{\varepsilon}, \varepsilon \in[0,1]$, точечной кривой $\gamma_{0}$ такой, что все кривые $\gamma_{\varepsilon}, \varepsilon>0$, являются регулярными, функция $\frac{\partial}{\partial \varepsilon} L\left(\gamma_{\varepsilon}(t), \dot{\gamma}_{\varepsilon}(t)\right)$ непрерывна по совокупности переменных $(t, \varepsilon)$ в области определения (это условие будем назьвать условием согласованности).

Примером квазирегулярного не всюду гладкого лагранжиана является лагранжиан, соответствующий функционалу длины на римановом многообразии. Более общий пример такого лагранжиана задается гладко зависящей от точки и не нулевого касательного вектора нормой на касательном пространстве к многообразию. Отметим, что строгая выпуклость нормы не предполагается.

Свойства, определяюшие квазирегулярньй лагранжиан, возникли при попытке понять, какие характеристики функционала длины приводят к наличию нетривиальных экстремалей. Из первого свойства вытекает, что регулярные ребра экстремальных сетей удовлетворяют стандартным уравнениям Эйлера-Лагранжа. Второе условие необходимо для того, чтобы функционал был корректно определен на сетях-следах (для этого необходимо равенство функционала нулю на точечных ребрах). Третье и четвертое свойства представляются необходимыми для использования стандартных приемов математического анализа, таких как дифференцирование интегралов, зависящих от параметра.

Следующее утверждение вычисляет производную по направлению от функционала $\psi_{L}$ с квазирегулярным лагранжианом.

УТВЕРЖДЕНИЕ 2.1. Пусть $L-$ квазирегулярный лагранжииан $u \psi_{L}-$ coответствующ,й классический вариачионный функиионал. Пусть $\gamma(t), t \in[a, b],-$ произвольная квазирегулярная кривая $и \gamma_{\varepsilon}, \varepsilon \in[0,1],-$ гладкая деформачия кривой $\gamma$ такая, что при $\varepsilon>0$ все кривые $\gamma_{\varepsilon}$ регулярны. Обозначим через $\eta(t)$ поле деформаиии $\gamma_{\varepsilon}$, m.е. $\eta(t)=d /\left.d \varepsilon\right|_{\varepsilon=0} \gamma_{\varepsilon}(t)$. Тогда если кривая $\gamma(t)$ регулярна, то выполнено равенство

$$
\left.\frac{d}{d \varepsilon}\right|_{\varepsilon=0} \psi_{L}\left(\gamma_{\varepsilon}\right)=\left.p(\gamma, \dot{\gamma})(\eta)\right|_{t=a} ^{t=b}+\int_{a}^{b}[L](\gamma, \dot{\gamma})(\eta) d t .
$$

В противном случае, т.е. если кривая $\gamma$ точечная, выполнено равенство

$$
\left.\frac{d}{d \varepsilon}\right|_{\varepsilon=0} \psi_{L}\left(\gamma_{\varepsilon}\right)=\int_{a}^{b} \zeta(L)(\gamma, 0) d t
$$

әде $\zeta=(\eta, \dot{\eta})$ - векторное поле вдоль кривой $t \mapsto(\gamma(t), 0)$ в TW. Если дополнительно предположить, что лагранжиан $L$ является гладким в окрестности точки $(x, 0)$, где $x=\gamma(t)$, то

$$
\left.\frac{d}{d \varepsilon}\right|_{\varepsilon=0} \psi_{L}\left(\gamma_{\varepsilon}\right)=\left.p(x, 0)(\eta)\right|_{t=a} ^{t=b}+\int_{a}^{b}[L](x, 0)(\eta) d t
$$

ДокАЗАТЕльство. Первое утверждение хорошо известно, см., например [2]. Докажем второе утверждение. Рассмотрим функцию

$$
f(t, \varepsilon)=L\left(\gamma_{\varepsilon}(t), \dot{\gamma}_{\varepsilon}(t)\right)
$$


где $t \in[a, b]$ и $\varepsilon \in[0,1]$. Частная производная $\frac{\partial}{\partial \varepsilon} f(t, \varepsilon)$ функции $f(t, \varepsilon)$ по параметру $\varepsilon$ равна $\zeta(L)(\gamma(t), \dot{\gamma}(t))$. Из условия квазирегулярности лагранжиана $L$ вытекает, что функция $\frac{\partial}{\partial \varepsilon} f(t, \varepsilon)$ непрерьвна по совокупности переменных. Поэтому

$$
\frac{d}{d \varepsilon} \int_{a}^{b} L\left(\gamma_{\varepsilon}(t), \dot{\gamma}_{\varepsilon}(t)\right) d t=\frac{d}{d \varepsilon} \int_{a}^{b} f(t, \varepsilon) d t=\int_{a}^{b} \frac{\partial}{\partial \varepsilon} f(t, \varepsilon) d t=\int_{a}^{b} \zeta(L)(\gamma(t), \dot{\gamma}(t)) d t
$$

что и требовалось.

Квазирегулярная (параметрическая) сеть Г назьвается әкстремальной по отношению к функционалу $\Psi_{L}$ с квазирегулярньп лагранжианом, если для произвольной деформации $\Gamma_{\varepsilon}, \varepsilon \in\left[0, \varepsilon_{0}\right]$, сети $\Gamma=\Gamma_{0}$ имеет место неравенство

$$
\left.\frac{d}{d \varepsilon}\right|_{\varepsilon=0} \Psi_{L}\left(\Gamma_{\varepsilon}\right) \geqslant 0
$$

ЗАмЕЧАниЕ. Чтобы не загромождать изложение, мы определяем экстремальность как неотрицательность производной по направлению. Сети, определяемые с помощью противоположного неравенства, тоже, конечно, следует назьвать экстремальными. Однако, мы, очевидно, не сужаем класса экстремальных сетей, так как одни получаются из других заменой знака лагранжиана.

Пусть $\Gamma: G \rightarrow W$ - произвольная квазирегулярная параметрическая сеть. Максимальные связные подграфы графа $G$, все ребра которых - точечные, назовем точечными компонентами графа $G$. Приведенные компоненты графа $G$ - это или его точечные компоненты, или его вершины, не инцидентные точечным ребрам. Соответствующие элементы параметрической сети назовем так же.

Для каждой приведенной компоненты $H \subset \Gamma$ обозначим через $E_{H}$ множество ребер сети $H$, через $I_{H}-$ множество смежных с $H$ ребер из $\Gamma \backslash H$, конечные вершины которых лежат в $H$, а через $O_{H}-$ множество смежных с $H$ ребер из $\Gamma \backslash H$, начальные вершины которых лежат в $H$. Отметим, что $I_{H}$ и $O_{H}$ могут пересекаться. Кроме того, объединение всех множеств $I_{H}$ и $O_{H}$ по всем приведенным компонентам $H$ сети $\Gamma$ совпадает с множеством всех регулярных ребер сети $Г$. Наконец, если $\gamma$ - ребро сети $\Gamma$, то через $\left[a_{\gamma}, b_{\gamma}\right]$ обозначим отрезок, параметризующий это ребро. Через $\partial H$ обозначим множество вершин из $H$, являюшихся граничными в сети Г. Через $\bar{\partial} H$ обозначим множество вершин из $H$, инцидентных регулярным ребрам сети Г. Далее, для каждой вершины $x \in \bar{\partial} H$ обозначим через $I_{H}(x)$ и $O_{H}(x)$ подмножества из $I_{H}$ и $O_{H}$ соответственно, состоящие из всех ребер, инцидентных $x$. Положим $N_{H}(x)=I_{H}(x) \cup O_{H}(x)$.

Удобно ввести следующее обозначение. Положим $p_{x}(\gamma)$ равньм $\pm p(\gamma, \dot{\gamma})$ в конщевой вершине $x$ ребра $\gamma$ сети $\Gamma$, где знак “плюс" выбирается, если $x$ - конечная вершина ребра $\gamma$, и знак "минус" - в противном случае.

Из утверждения 2.1 легко получается следующий результат.

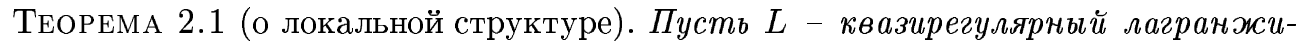
ан. Квазирегулярная параметрическая сеть $\Gamma: G \rightarrow W$ с граничей $\beta$ является әкстремалью функиионала $\Psi_{L}$, если и только если каждое регулярное ребро $\gamma:\left[a_{\gamma}, b_{\gamma}\right] \rightarrow W$ сети $\Gamma$ есть әкстремаль функиионала $\psi_{L}$, m.e. $[L](\gamma, \dot{\gamma})=0, u$ 
для каждой приведенной компоненты $H: G_{H} \rightarrow W$ сети $\Gamma$ и любой гладкой сети $\eta: G_{H} \rightarrow T W$, дде $\pi \circ \eta=\left.\Gamma\right|_{G_{H}}$ и $\eta\left(\partial G_{H}\right) \subset W_{0}$, следующее выражение неотричательно:

$$
\begin{gathered}
\left.\sum_{\gamma \in I_{H}} p(\gamma, \dot{\gamma})\left(\eta_{\gamma}\right)\right|_{t=b_{\gamma}}-\left.\sum_{\gamma \in O_{H}} p(\gamma, \dot{\gamma})\left(\eta_{\gamma}\right)\right|_{t=a_{\gamma}}+\sum_{\gamma \in E_{H}} \int_{a_{\gamma}}^{b_{\gamma}} \zeta_{\gamma}(L)(\gamma, \dot{\gamma}) d t \\
=\sum_{x \in \bar{\partial} H}\left[\sum_{\gamma \in N_{H}(x)} p_{x}(\gamma)\right](\eta(x))+\sum_{\gamma \in E_{H}} \int_{a_{\gamma}}^{b_{\gamma}} \zeta_{\gamma}(L)(\gamma, \dot{\gamma}) d t
\end{gathered}
$$

где $\eta_{\gamma}-$ ребро $\left.\eta\right|_{\left[a_{\gamma}, b_{\gamma}\right]}$ сети $\eta$, a $\zeta_{\gamma}=\left(\eta_{\gamma}, \dot{\eta}_{\gamma}\right)$ - векторное поле вдоль кривой $(\gamma, \dot{\gamma})$ в $T W$.

Определим поднятие параметрической сети $\Gamma: G \rightarrow W$ в касательное расслоение как множество кривых $\{(\gamma(t), \dot{\gamma}(t))\}$ по всем ребрам $\gamma$ сети $\Gamma$.

СлЕДСТВИЕ 2.1. Пусть в предположениях теоремы 2.1 лагранжиан $L$ является гладким в окрестности поднятия сети (например, это имеет место, если сеть Г регулярна). Тогда квазирегулярная параметрическая сеть $\Gamma: G \rightarrow W c$ граничей $\beta$ является әкстремалью функиионала $\Psi_{L}$, если и только если каждое ребро $\gamma:\left[a_{\gamma}, b_{\gamma}\right] \rightarrow W$ сети $\Gamma$ есть әкстремаль функиионала $\psi_{L}$, m.е. $[L](\gamma, \dot{\gamma})=0$, и для каждой подвижной вершины $x$ сети Г имеет место равенство

$$
\sum_{\gamma} p_{x}(\gamma)=0
$$

где сумма берется по всем ребрам $\gamma$, иниидентным вериине $x$.

3. Локальная структура экстремальных сетей. В этом пунктемы рассмотрим экстремальные сети-следы и изучим их локальную структуру. Оказьвается, нетривиальность локальной структуры связана в первую очередь с негладкостью лагранжиана $L$, соответствующего классическому вариационному функционалу.

Пусть $L-$ квазирегулярный лагранжиан. Тогда функционал $\Psi_{L}$, заданньй на параметрических квазирегулярных сетях, порождает функционал на множестве соответствующих сетей-следов, которьй мы обозначим той же буквой. Отметим, что канонические представители таких сетей являются регулярньми сетями. Мы начнем с разбора ситуации, когда лагранжиан $L$ является гладким.

3.1. Гладкие лагранжианы. Пусть лагранжиан $L$ гладкий на всем $T W$. Предположим также, что $L$ равен нулю на нулевом сечении $W_{0}$. Тогда $L$, в частности, является квазирегулярным лагранжианом. Имеет место следуюший результат.

ТЕОРема 3.1. Сеть $\Upsilon$ является әкстремалью для функиионала $\Psi_{L}$ с гладким лагранжианом $L$, равным нулю на нулевом сечении $W_{0} \subset T W$, если и только если выполняется следуюшие свойства:

1) каждое ребро ү сети $\Upsilon$ является әкстремалью функиионала $\psi_{L}$;

2) для любой вериины $x$ сети $\Upsilon$ и любого ребра $\gamma$, иниидентного $x$, имеют место равенства $p(x, 0)=0 u p_{x}(\gamma)=0$. 
ДоКАЗАТЕЛЬСТВО. По определению сеть $\Upsilon$ является экстремальной сетью, если и только если любой ее представитель Г является экстремальной сетью для соответствующего функционала на параметрических сетях. В частности, канонический представитель $Г$ сети $\Upsilon$ удовлетворяет условия теоремы 2.1 , поэтому его ребра - экстремали функционала $\psi_{L}$, что доказьвает необходимость первого условия.

Аналогично, воспользовавшись утверждением 2.1, можно показать, что каждое точечное ребро произвольного представителя $\Gamma$ сети $\Upsilon$ является экстремалью функционала $\psi_{L}$, т.е. удовлетворяет уравнению Эйлера-Лагранжа.

Далее, пусть $\gamma$ - произвольное ребро сети $\Upsilon$, инщидентное вершине $x$. Рассмотрим представитель $Г$ сети $\Upsilon$ такой, что

1) приведенная компонента вершины $x$ сети $\Gamma$ состоит из одного ребра $\gamma^{\prime}$;

2) ребро $\gamma^{\prime}$ инцидентно вершине $x^{\prime}$, имеющей в Г степень 2 и инцидентной в свою очередь ребру $\gamma$.

Построенная параметрическая сеть Г является экстремальной, поэтому в силу следствия 2.1, примененного к вершине $x^{\prime}$, имеет место равенство $p_{x^{\prime}}(\gamma) \pm p\left(x^{\prime}, 0\right)=0$, где знак выбирается в зависимости от ориентации ребра $\gamma^{\prime}$. Так как эта ориентация может быть выбрана любой и так как $p_{x^{\prime}}(\gamma)=p_{x}(\gamma)$, отсюда вытекает, что

$$
p_{x}(\gamma)=p\left(x^{\prime}, 0\right)=-p\left(x^{\prime}, 0\right)=p(x, 0)=0
$$

что и требовалось.

Обратное утверждение немедленно вытекает из следствия 2.1, так как все импульсы равны нулю по условию. Теорема доказана.

СледСТВИЕ 3.1. Пусть $\Upsilon$ - әкстремальная сеть для функиионала $\Psi_{L}$ с гладким лагранжиианом $L$, равным нулю на нулевом сечении $W_{0} \subset T W$. Тогда каждое ребро сети $\Upsilon$ есть экстремаль функиионала $\psi_{L}$ по отношению к произвольным деформачиям, и кажсдая точечная кривая, образ которой совпадает с произвольной вершиной сети $\Upsilon$, также является әкстремалью функиионала $\psi_{L}$ по отношению $\kappa$ любым деформачиям. Другими словами, каждая әкстремальная сеть функционала $\Psi_{L}$ является тривиально әкстремальной.

СлЕДСТВИЕ 3.2. Пусть $\Upsilon$ - әкстремальная сеть для функиионала $\Psi_{L}$ с гладким лагранжианом $L$, равным нулю на нулевом сечении $W_{0} \subset T W$. Пусть $x-$ вершина сети $\Upsilon$, а $\gamma_{1}, \ldots, \gamma_{k}-$ инцидентные $x$ ребра сети $\Upsilon$. Обозначим через $\xi_{i}$, $i=1, \ldots, k$, вектор скорости ребра $\gamma_{i}$ в вершине $x$. Тогда каждая точка $\left(x, \xi_{i}\right), a$ также точка $(x, 0)$, является стационарной точкой ограничения лагранжиана $L$ на $T_{x} W$.

СлЕДСТВИЕ 3.3. Пусть $L$ - гладкий лагранжиан, равный нулю на нулевом сечении $W_{0} \subset T W$. Если лагранэсиан $L$, ограниченный на $T_{x} W$, не имеет стационарных точек вне $0 \in T_{x} W$ для любого $x \in W$, то кажсдая әкстремальная сеть функиионала $\Psi_{L}-$ точечная.

ПРИМЕР. Пусть $L(x, \xi)=\langle\xi, \xi\rangle-$ лагранжиан, соответствующий функционалу энергии на римановом многообразии $W$. Из следствия 3.3 вытекает, что в классеследов этот функционал не имеет экстремалей, отличных от точечных. 
ПРИмеР. Пусть $L(x, \xi)=T(\xi)-U(x)$ - лагранжиан, соответствующий функционалу, описывающему движение материальной точки в поле консервативных сил с потенциалом $U(x)$ на римановом многообразии $W$. Из следствия 3.3 вытекает, что в классе следов этот функционал не имеет экстремалей, отличных от точечных.

СлЕДСТВИЕ 3.4. Пусть $L$ - гладкий лагранжиан, равный нулю на нулевом сечении $W_{0} \subset T W$. Предположим, что решение задачи Коши для уравнения Эйлера-Лагранжа с лагранэсианом $L$ локально единственно. Тогда если лагранжииан $L$, ограниченный на $T_{x} W$, имеет не более двух стачионарных точек для любого $x \in W$, то каждая әкстремальная сеть функиионала $\Psi_{L}$ - либо точка, либо кривая (канонический представитель имеет лишь вершины степени 1 и 2).

3.2. Квазирегулярные лагранжианы. Вернемся к случаю квазирегулярных лагранжианов общего вида.

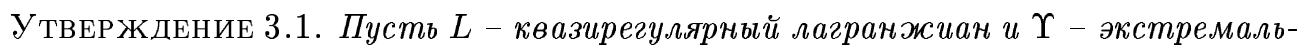
ная сеть для функиионала $\Psi_{L}$. Тогда $\zeta(L)(x, 0) \geqslant 0$ для любой вершины $x \in \Upsilon u$ любого вектора $\zeta \in T_{(x, 0)} T W$.

ДокАЗАТЕЛЬСТво. Предположим противное, а именно, пусть в некоторой вершине $x$ для некоторого $\zeta_{0} \in T_{(x, 0)} T W$ имеет место неравенство $\zeta_{0}(L)(x, 0)<0$. Рассмотрим представитель $\Gamma$ сети $\Upsilon$, прообраз вершины $x$ которого состоит из одного ребра $\gamma:[0, \delta] \rightarrow x$, инцидентного вершине степени 1 , которая соответствует концу $\delta$ параметризующего отрезка $[0, \delta]$.

Фиксируем в окрестности точки $x$ на многообразии $W$ произвольные локальные координаты $x^{i}$. Тем самым, у нас заданы локальные координаты $\left(x^{i}, \xi^{j}\right)$ на расслоении $T W$. Пусть в этих координатах вектор $\zeta_{0}$ имеет вид $\zeta=\alpha^{i} \partial_{x^{i}}+\beta^{i} \partial_{\xi^{i}}$. Положим $\alpha=\left(\alpha^{1}, \ldots, \alpha^{n}\right)$ и $\beta=\left(\beta^{1}, \ldots, \beta^{n}\right)$, где $n=\operatorname{dim} W$. Построим деформацию параметрической сети $\Gamma$, постоянную на всех ее ребрах, кроме ребра $\gamma$, а на ребре $\gamma$ зададим ее так: $\gamma_{\varepsilon}(t)=x+\varepsilon(\alpha+t \beta)$. Тогда поле $\eta(t)$ этой деформации имеет вид $\eta(t)=\alpha+t \beta$, а соответствуюшее поле $\zeta(t)$ равно $(\eta(t), \dot{\eta}(t))=(\alpha+t \beta, \beta)$. В частности, $\zeta(0)=\zeta_{0}$. Следовательно, выбрав $\delta$ достаточно мальм и воспользовавшись условием согласованности, получим:

$$
\int_{0}^{\delta} \zeta(L) d t<0 .
$$

Последнее противоречит критичности сети $\Gamma$, см. теорему 2.1. Утверждение доказано.

УТВЕРЖДЕНИЕ 3.2. Пусть $L-$ квазирегулярный лагранэсиан $u \Upsilon-$ әкстремальная сеть для функиионала $\Psi_{L}$. Тогда в каждой подвижной вершине $x$ сети $\Upsilon$ сумма импульсов $p_{x}(\gamma)$ по всем иниидентным $x$ ребрам $\gamma$ из $\Upsilon$ равна нулю.

ДокАЗАТЕЛЬСТво. Это так в силу следствия 2.1, поскольку канонический представитель является регулярной критической сетью.

Обозначим через $V(T W)$ подрасслоение в расслоении $T(T W) \rightarrow T W$, каждьй слой которого состоит из всех векторов, касательных к слою касательного расслоения $T W \rightarrow W$. Обозначим через $H\left(W_{0}\right)$ распределение касательных плоскостей $\mathrm{k}$ нулевому сечению $W_{0} \subset T W$. В каждой точке $x$ из $W$ касательное пространство $T_{(x, 0)} T W$ раскладывается в прямую сумму подпространств $V_{(x, 0)} T W$ и $H_{(x, 0)} W_{0}$. Плоскости $H_{(x, 0)} W_{0}$ назовем горизонтальными плоскостями. 
Отметим, что каждьй вектор $\zeta \in T_{(x, 0)} T W$ может быть однозначно разложен в сумму своих вертикальной $\zeta_{v} \in V_{(x, 0)} T W$ и горизонтальной $\zeta_{h} \in H_{(x, 0)} W_{0}$ составляющих.

Будем говорить, что квазирегулярньй лагранжиан $L$ дифферениируем вдоль базы $W$, если для каждого $x \in W$ и каждого вектора $\zeta \in T_{(x, 0)} T W$ имеет место равенство

$$
\zeta(L)=\zeta_{v}(L)+\zeta_{h}(L)
$$

причем $\zeta_{h}(L)$ - линейная функция от $\zeta_{h} \in H_{(x, 0)} W_{0}$.

УТВЕРЖДЕНИЕ 3.3. Пусть $L$ - квазирегулярный лагранэсиан $u \Upsilon-$ экстремальная сеть для функционала $\Psi_{L}$. Предположим, что лагранжиан $L$ дифференцируем вдоль базы. Тогда для каждой вершины $x$ сети $\Upsilon$ ограничение функиии $\zeta(L)$ на горизонтальную плоскость $H_{(x, 0)} W_{0}$ равно нулю.

ДокАЗАТЕльСтво. В силу утверждения 3.1 имеем $\zeta(L)(x, 0) \geqslant 0$ для любого $\zeta \in$ $T_{(x, 0)} T W$, в частности, для всех $\zeta$ из горизонтальной плоскости $H_{(x, 0)} W_{0}$. Из дифференцируемости лагранжиана $L$ вдоль базы вытекает, что ограничение функции $\zeta(L)(x, 0)$ на плоскость $H_{(x, 0)} W_{0}$ есть линейная функция, которая тем самым равна нулю. Доказательство закончено.

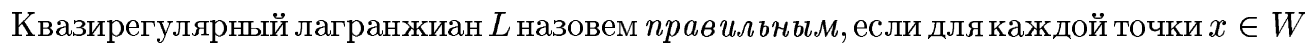
функция $f(\zeta)=\zeta(L)(x, 0)$ на $T_{(x, 0)} T W$ является гладкой вне $\zeta=0$.

Вершину $x$ следа $\Upsilon$ (соответствующую вершину канонического представителя для $\Upsilon$ ) назовем свободной, если импульсы $p_{x}(\gamma)$ инцидентных ей ребер $\gamma$ равны нулю.

Напомним, что существует канонический изоморфизм $\mu$ между касательными пространствами $T_{x} W$ к многообразию $W$ и соответствуюшими вертикальными пространствами $V_{(x, 0)} T W$. Этот изоморфизм определяется так. Пусть $\xi$ - произвольньй вектор из $T_{x} W$. Рассмотрим гладкую кривую $\gamma(t)=(x, t \xi)$ в $T W$, выходящую из точки $\gamma(0)=(x, 0)$. Вектор скорости $\dot{\gamma}(0)$ по определению лежит в $V_{(x, 0)} T W$. Изоморфизм $\mu$ сопоставляет вектору $\xi$ вектор $\dot{\gamma}(0)$.

УТВЕРЖДЕНИЕ 3.4. Пусть $\Upsilon$ - сеть-след, являющаяся әкстремалью функиионала $\Psi_{L}$, где $L$ - правильный квазирегулярный лагранжиан, дифференцируемый

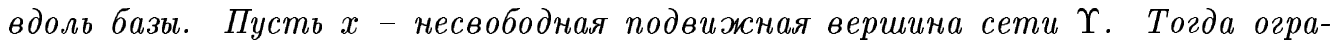
ничение функиии $f(\zeta)=\zeta(L)(x, 0)$ на $V_{(x, 0)} T W \backslash\{0\}$ положительно. При әтом $\zeta(L)(x, 0)=\zeta_{v}(L)(x, 0)$.

ДокАЗАТЕЛЬСтво. Из утверждения 3.3 следует равенство $\zeta(L)(x, 0)=\zeta_{v}(L)(x, 0)$. Кроме того, из утверждения 3.1 следует, что $\zeta(L)(x, 0) \geqslant 0$. Таким образом, для доказательства утверждения достаточно проверить, что $\zeta(L)(x, 0)$ не может равняться нулю для вертикальных ненулевых векторов $\zeta \in V_{(x, 0)} T W$.

Предположим противное, т.е. пусть в некоторой несвободной вершине $x$ сети $\Upsilon$ для некоторого вертикального вектора $\zeta_{0} \neq 0$ имеет место равенство $\zeta_{0}(L)(x, 0)=0$. Так как вершина $x$ несвободна, существует такое инцидентное $x$ ребро $\gamma$, что его импульс $p_{x}(\gamma)$ отличен от нуля. Рассмотрим представитель $Г$ сети $\Upsilon$, для которого прообраз вершины $x$ состоит из одного ребра $\gamma^{\prime}:[-a, a] \rightarrow x$. При этом одна из инцидентных $\gamma^{\prime}$ вершин, которую мы обозначим через $x^{\prime}$, инцидентна ребру $\gamma$, а другая, обозначаемая через $x^{\prime \prime},-$ всем остальньм ребрам $\gamma_{1}, \ldots, \gamma_{k}$, инцидентньм вершине $x$ в $\Upsilon$.

Положим $\mu^{-1}\left(\zeta_{0}\right)=\nu_{0}$. Построим деформацию сети $\Gamma^{\prime}$, оставляющую на месте все вершины сети $\Gamma^{\prime}$, кроме вершин ребра $\gamma^{\prime}$. 
Предположим сначала, что $p_{x}(\gamma)\left(\nu_{0}\right) \neq 0$. Если $p_{x}(\gamma)\left(\nu_{0}\right)>0$, то ребро $\gamma^{\prime}$ выберем так, чтобы точка - $a$ из параметризующего отрезка $[-a, a]$ соответствовала $x^{\prime}$. Если же $p_{x}(\gamma)\left(\nu_{0}\right)<0$, то будем предполагать, что $-a$ соответствует вершине $x^{\prime \prime}$. Для определенности будем предполагать, что имеет место вторая возможность.

Пусть в окрестности точки $(x, 0)$ касательного расслоения $T W$ заданы локальные координаты $\left(x^{i}, \xi^{j}\right)$, порожденные некоторьми координатами $x^{i}$ на $W$. Деформацию на ребре $\gamma^{\prime}$ зададим так: $\gamma_{\varepsilon}^{\prime}(t)=\varepsilon \nu_{0}(t+a) /(2 a)$, где $\varepsilon \in\left[0, \varepsilon_{0}\right]$. Тогда поле деформации $\eta(t)$ ребра $\gamma^{\prime}$ имеет вид: $\eta(t)=\nu_{0}(t+a) /(2 a)$, а его поднятие $\zeta(t)$ равно

$$
(\eta(t), \dot{\eta}(t))=\left(\frac{t+a}{2 a} \nu_{0}, \frac{1}{2 a} \nu_{0}\right) \text {. }
$$

Вертикальная составляющая поля $\zeta(t)$, равная $\dot{\eta}(t)$, имеет вид $\nu_{0} /(2 a)$, откуда $\zeta_{v}(L)(x$, $0)=0$.

С другой стороны, $\eta(-a)=0$, а $\eta(a)=\nu_{0}$, поэтому первая вариация функционала $\Psi_{L}$ для рассматриваемой деформации сети $Г$ равна

$$
p_{x}(\gamma)\left(\nu_{0}\right)+\int_{-a}^{a} \zeta_{v}(L)(x, 0) d t=p_{x}(\gamma)\left(\nu_{0}\right)<0
$$

противоречие. Осталось заметить, что суммарньй импульс $p$ ребер, инцидентных вершине $x^{\prime \prime}$ сети $\Gamma$, противоположен импульсу $p_{x}(\gamma)$. Поэтому если $p_{x}(\gamma)\left(\nu_{0}\right)$ положительно, то $p\left(\nu_{0}\right)$ отрищательно, и применимы те же рассуждения.

Пусть теперь $p_{x}(\gamma)\left(\nu_{0}\right)=0$. Так как $p_{x}(\gamma) \neq 0$, существует такое направление $\theta \in T_{x} W$, что $p_{x}(\gamma)(\theta)<0$. Рассмотрим функцию $A(\delta)=\left(\nu_{0}+\delta \theta\right)(L)(x, 0)$. По определению правильного лагранжиана функция $A$ гладкая при достаточно мальх $\delta$. Более того, точка $\delta=0$ является точкой минимума функции $A$. Действительно, $A(0)=0$ в силу выбора $\nu_{0}$, а по утверждению $3.1 A(\delta) \geqslant 0$. Поэтому

$$
A(\delta)=o(\delta), \quad \delta \rightarrow 0
$$

так как 0 - внутренняя точка интервала изменения параметра $\delta$.

Рассмотрим деформацию сети $\Gamma$, построенную так же, как в первом случае, но при использовании направления $\nu_{0}+\delta \theta$ вместо направления $\nu_{0}$. Запишем формулу первой вариации:

$$
p_{x}(\gamma)\left(\nu_{0}+\delta \theta\right)+\frac{1}{2 a} \int_{-a}^{a} A(\delta) d t=\delta\left(p_{x}(\gamma)(\theta)+o(1)\right), \quad \delta \rightarrow 0
$$

Поэтому при достаточно малых $\delta>0$ первая вариация отрицательна, что противоречит критичности сети $Г$. Доказательство закончено.

СлЕдСТВИЕ 3.5. Пусть $\Upsilon$ - сеть-след, являющаяся әкстремалью функиионала $\Psi_{L}$, где L - правильный квазирегулярный лагранжиан, дифференцируемый вдоль

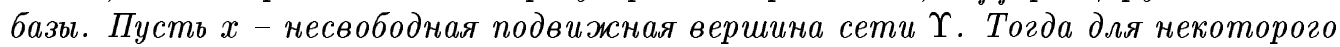
числа $c(x)>0$, зависящего только от точки $x$, июбого $\eta \in T_{x} W$ выполняется неравенство

$$
\lim _{\varepsilon \rightarrow 0+} \frac{L(x, \varepsilon \eta)}{\varepsilon} \geqslant c(x)\|\eta\| .
$$

$B$ частности, ограничение функции $L$ на любое линейное подпространство пространства $V_{(x, 0)} T W$ не дифференцируемо в нуле.

Докажем еще одно свойство критических следов. 
УТВЕРЖДЕНИЕ 3.5. Пусть $\Upsilon$ - сеть-след, являющаяся әкстремалью функиионала $\Psi_{L}$, где $L$ - правильный квазирегулярный лагранжиан, дифференцируемый вдоль базы. Тогда в каждой вершине $x$ сети $\Upsilon$ для любых иниидентных $x$ ребер $\gamma_{1}, \ldots, \gamma_{k}$ и любого вектора $\eta \in T_{x} W$ выполнено следующее условие:

$$
\sum_{i=1}^{k} p_{x}\left(\gamma_{i}\right)(\eta)+\mu(\eta)(L)(x, 0) \geqslant 0
$$

ДокАЗАТЕЛЬСтво. Разобьем все ребра сети $\Upsilon$, инцидентные вершине $x$, на два класса, отнеся к первому ребра $\gamma_{1}, \ldots, \gamma_{k}$, а ко второму - все остальные ребра. Рассмотрим представитель $Г$ сети $\Upsilon$, для которого прообраз вершины $x$ состоит из одного ребра $\gamma:[-a, a] \rightarrow x$. При этом одна из инцидентных $\gamma$ вершин, которую мы обозначим через $x^{\prime}$, инцидентна ребрам $\gamma_{1}, \ldots, \gamma_{k}$, а другая, обозначаемая через $x^{\prime \prime},-$ всем остальным ребрам, инцидентным вершине $x$ в $\Upsilon$. Без ограничения общности будем считать, что вершине $x^{\prime}$ соответствует конец $a$ параметризуюшего отрезка $[-a, a]$.

Фиксируем на $W$ в окрестности точки $x$ произвольные локальные координаты $x^{i}$, и без ограничения общности предположим, что в этих координатах $x=0$.

Рассмотрим деформацию $\Gamma_{\varepsilon}$ сети $\Gamma$, при которой все вершины сети $\Gamma$, кроме вершины $x^{\prime}$, остаются на месте, а вершина $x^{\prime}$ равномерно движется со скоростью $\nu: x^{\prime}(\varepsilon)=\varepsilon \nu$. При этом ребро $\gamma$ деформируется линейно: $t \mapsto \varepsilon \nu(t+a) /(2 a)$ для каждого $t \in[-a, a]$. Тогда поле деформации $\eta(t)$ вдоль ребра $\gamma$ имеет вид $\eta(t)=\nu(t+a) /(2 a)$, откуда $\dot{\eta}(t)=\nu /(2 \alpha)$. Как всегда, положим $\zeta(t)=(\eta(t), \dot{\eta}(t))$. Запишем условие неотрицательности первой вариации для такой деформации, учитьвая, что $\zeta(L)=\zeta_{v}(L)=\dot{\eta}(L)$ в силу утверждения 3.3. Имеем:

$$
\left(\sum_{i=1}^{k} p_{x}\left(\gamma_{i}\right)\right)(\nu)+\frac{1}{2 a} \int_{-a}^{a} \nu(L)(x, 0) d t=\left(\sum_{i=1}^{k} p_{x}\left(\gamma_{i}\right)\right)(\nu)+\nu(L)(x, 0) \geqslant 0 .
$$

Доказательство закончено.

Следующая теорема является критерием критичности сети-следа по отношению к функционалу, заданному правильным квазирегулярньп лагранжианом, дифференцируемьм вдоль базы.

Теорема 3.2. Сеть-след $\Upsilon$ является әкстремалью функиионала $\Psi_{L}$ с правильным квазирегулярным лагранжианом L, дифферениируемым вдоль базы, если $и$ только если выполняются следуюшие условия:

1) каждое ребро ү сети $\Upsilon$ является әкстремалью функиионала $\psi_{L}$;

2) в каждой вершине $x$ сети $\Upsilon$ для любых иниидентных х ребер $\gamma_{1}, \ldots, \gamma_{k} u$ любого вектора $\eta \in T_{x} W$ выполнено условие

$$
\sum_{i=1}^{k} p_{x}\left(\gamma_{i}\right)(\eta)+\mu(\eta)(L)(x, 0) \geqslant 0
$$

3) в каждой подвижной вершине сети $\Upsilon$ сумма импульсов инцидентных этой вершине ребер равна нулю;

4) в каждой неподвижной вершине $x$ сети $\Upsilon$ для любого $\zeta \in V_{(x, 0)} W$ имеет место неравенство $\zeta(L)(x, 0) \geqslant 0$; отметим, что если записать $\zeta$ в виде $\mu(\eta), \eta \in T_{x} W$, то условие этого пункта перепишется так: $\mu(\eta)(L)(x, 0) \geqslant 0$. 
ДокАЗАТЕльство. Пусть след $\Upsilon$ экстремален. Тогда канонический представитель сети $\Upsilon$ - регулярная сеть, и по следствию 2.1 все его ребра - экстремали функционала $\psi_{L}$. Справедливость остальных свойств сети $\Upsilon$ доказана в утверждениях 3.1-3.5.

Перейдем к доказательству достаточности. Предположим, что сеть $\Upsilon$ удовлетворяет условиям 1)-4) теоремы. Пусть $\Gamma: G \rightarrow W$ - произвольньй представитель сети $\Upsilon$ и $\left[\Gamma_{\varepsilon}\right]$ - некоторая деформация следа $\Upsilon$. Мы должны показать, что первая вариация функционала $\Psi_{L}$ для этой деформации неотрицательна. Так как первая вариация функционала $\Psi_{L}$ раскладьвается в сумму выражений, соответствующих приведенньгм компонентам параметрической сети $Г$, достаточно проверить неотрицательность первой вариации в предположении, что деформация $\Gamma_{\varepsilon}$ оставляет на месте все приведенные компоненты сети $\Gamma$, кроме одной, которую мы обозначим через $H$. Обозначим через $x$ соответствующую $H$ вершину из $\Upsilon$.

Пусть $\bar{\partial} H=\left\{x_{1}, \ldots, x_{k}\right\}$ - множество вершин сети $H$, инцидентных регулярным ребрам из $Г$. Обозначим через $E^{i}$ множество невырожденных ребер сети $\Gamma$, инцидентных вершине $x_{i}$, а через $p_{x}\left(E^{i}\right)$ - суммарньй импульс ребер из $E^{i}$ в вершине $x$.

Нам понадобится следующая лемма.

Лемма 3.1. Если $x$ - подвижная вериина сети $\Upsilon$, то для любого $\eta \in T_{x} W$ имеет место неравенство $\mu(\nu)(L)(x, 0) \geqslant 0$.

ДокАЗАТЕльство. Действительно, воспользуемся условием 2) для случая, когда множество $\left\{\gamma_{1}, \ldots, \gamma_{k}\right\}$ ребер совпадает с множеством всех ребер из $\Upsilon$, инцидентных $x$. Для произвольного вектора $\eta$ имеем:

$$
\sum_{i=1}^{\operatorname{deg} x} p_{x}\left(\gamma_{i}\right)(\eta)+\mu(\eta)(L)(x, 0) \geqslant 0
$$

Так как $x$ - подвижная вершина, то сумма в левой части этого неравенства равна нулю в силу свойства 3 ), откуда и вытекает требуемое. Лемма доказана.

Отметим, что в силу условия 4) и леммы 3.1 достаточно ограничиться случаем, когда сеть $H$ является деревом.

Пусть $\gamma$ - произвольное ребро сети $H$. Обозначим через $\left[a_{\gamma}, b_{\gamma}\right]$ параметризующий это ребро отрезок, и пусть $x_{a}$ и $x_{b}$ - вершины ребра $\gamma$, соответствующие $a_{\gamma}$ и $b_{\gamma}$. Если мы разрежем дерево $H$ по ребру $\gamma$, то сеть $H$ распадется на две компоненты $H_{1}$ и $H_{2}$, где через $H_{1}$ обозначена компонента, содержащая вершину $x_{a}$. Разобьем множество невырожденных ребер сети $\Gamma$, инцидентных $H$, на два класса $E_{1}^{\gamma}$ и $E_{2}^{\gamma}$, отнеся к классу $E_{i}^{\gamma}$ ребра, смежные с компонентой $H_{i}$.

Фиксируем в окрестности точки $x$ на многообразии $W$ локальные координаты. Тогда в соответствуюших координатах на $T(T W)$ изоморфизм $\mu$ задается единичной матрицей. Поэтому ниже мы будем опускать его в формулах.

Обозначим через $\eta_{\gamma}(t), t \in\left[a_{\gamma}, b_{\gamma}\right]$, поле деформации $\Gamma_{\varepsilon}$ на ребре $\gamma$, а через $p_{x}\left(E_{i}^{\gamma}\right)-$ сумму импульсов ребер из $E_{i}^{\gamma}$ в вершине $x$. Тогда из условия 2$)$ вытекает, что для каждого $t \in\left[a_{\gamma}, b_{\gamma}\right]$ имеют место неравенства

$$
p_{x}\left(E_{i}^{\gamma}\right)\left(\dot{\eta}_{\gamma}(t)\right)+\dot{\eta}_{\gamma}(t)(L)(x, 0) \geqslant 0, \quad i=1,2 .
$$

Проинтегрировав эти неравенства по $t$ от $a_{\gamma}$ до $b_{\gamma}$, получим:

$$
p_{x}\left(E_{i}^{\gamma}\right)\left(\eta_{\gamma}\left(b_{\gamma}\right)\right)-p_{x}\left(E_{i}^{\gamma}\right)\left(\eta_{\gamma}\left(a_{\gamma}\right)\right)+\int_{a_{\gamma}}^{b_{\gamma}} \dot{\eta}_{\gamma}(t)(L)(x, 0) d t \geqslant 0, \quad i=1,2 .
$$


Предположим, что вершина $x$ сети $\Upsilon$ подвижна. Тогда в силу условия 3$) p_{x}\left(E_{1}^{\gamma}\right)+$ $p_{x}\left(E_{2}^{\gamma}\right)=0$, поэтому

$$
\begin{aligned}
& p_{x}\left(E_{2}^{\gamma}\right)\left(\eta_{\gamma}\left(b_{\gamma}\right)\right)-p_{x}\left(E_{2}^{\gamma}\right)\left(\eta_{\gamma}\left(a_{\gamma}\right)\right)+\int_{a_{\gamma}}^{b_{\gamma}} \dot{\eta}_{\gamma}(t)(L)(x, 0) d t \\
& \quad=p_{x}\left(E_{2}^{\gamma}\right)\left(\eta_{\gamma}\left(b_{\gamma}\right)\right)+p_{x}\left(E_{1}^{\gamma}\right)\left(\eta_{\gamma}\left(a_{\gamma}\right)\right)+\int_{a_{\gamma}}^{b_{\gamma}} \dot{\eta}_{\gamma}(t)(L)(x, 0) d t \geqslant 0 .
\end{aligned}
$$

Пусть $y$ - одна из двух инцидентных $\gamma$ вершин. Если $y=x_{a} \in H_{1}$, то положим $p_{y}^{\gamma}=$ $p_{x}\left(E_{1}^{\gamma}\right)$, а $\bar{p}_{y}^{\gamma}=p_{x}\left(E_{2}^{\gamma}\right) ;$ в противном случае положим $p_{y}^{\gamma}=p_{x}\left(E_{2}^{\gamma}\right)$, a $\bar{p}_{y}^{\gamma}=p_{x}\left(E_{1}^{\gamma}\right)$.

Запишем неравенство $(2)$ для каждого ребра $\gamma$ из $H$ и сложим эти неравенства. Полученная сумма распадается на две: сумма $\sum_{2}$ интегралов по всем ребрам из $H$ (эта сумма совпадает с интегральной частью формулы первой вариации для деформации $\left.\Gamma_{\varepsilon}\right)$ и сумма $\sum_{1}$ импульсов. Мы покажем, что эта сумма импульсов совпадает с неинтегральной частью формулы первой вариации для деформации $\Gamma_{\varepsilon}$, что и завершит разбор случая подвижной вершины $x$. Для этого сгруппируем слагаемые из суммы $\sum_{1}$, собрав вместе импульсы, примененные к значению поля деформации $\eta$ в одной и той же вершине $y$ сети $H$, и обозначим их сумму через $\sum_{y}$.

Пусть $y \in \bar{\partial} H$, т.е. $y=x_{i}$ для подходящего $i$, и пусть $d$ - это степень вершины $y$ в $H$. Обозначим через $f_{1}, \ldots, f_{d}$ инцидентные $y$ ребра из $H$. Тогда сумма $\sum_{y}$ имеет вид $\sum_{j} p_{y}^{f_{j}}$, или

$$
\sum_{y}=-\sum_{j} \bar{p}_{y}^{f_{j}} .
$$

Осталось заметить, что в правой части стоит выражение

$$
-\sum_{j} \bar{p}_{y}^{f_{j}}=-\sum_{j \neq i} p_{x}\left(E^{j}\right)=p_{x}\left(E^{i}\right) .
$$

Последнее равенство имеет место в силу предположения 3$)$. Таким образом, $\sum_{x_{i}}=$ $p_{x}\left(E^{i}\right)$.

Пусть теперь $y \notin \bar{\partial} H$. Тогда $\sum_{y}=\sum_{\gamma} p_{y}^{\gamma}$, где суммирование берется по всем ребрам $\gamma$ из $H$, инцидентньм $y$. Так как вершина $x$ подвижная, то $p_{y}^{\gamma}+\bar{p}_{y}^{\gamma}=0$ по предположению. Поэтому $\sum_{y}=-\sum_{\gamma} \bar{p}_{y}^{\gamma}$. Легко видеть, что последняя сумма равна сумме импульсов в вершине $x$ всех ребер из $\Upsilon$, инцидентных $x$, и, значит, равна нулю. Тем самым, случай подвижной вершины $x$ полностью разобран.

Пусть теперь $x$ - неподвижная вершина сети $\Upsilon$. Среди вершин сети $H$ в этом случае необходимо имеются граничные. Напомним, что в граничной вершине $x$ сумма импульсов ребер сети $\Upsilon$, инцидентных $x$, не предполагается равной нулю. Обозначим эту сумму через $p$. Пусть $y$ - неподвижная вершина из $H$. Как и выше, обозначим через $\eta$ поле деформации $\Gamma_{\varepsilon}$, и пусть $\eta_{y}$ - значение поля $\eta$ в вершине $y$. Так как $y$ неподвижна, то $\eta_{y}=0$.

Запишем формулу первой вариации для деформации $\Gamma_{\varepsilon}$ и добавим к ней нулевое слагаемое $-p\left(\eta_{y}\right)$. Для завершения доказательства теоремы достаточно показать, что полученное выражение неотрицательно. Обозначим через $p_{y}$ сумму импульсов в вершине $y$ невырожденных ребер сети $\Gamma$, инцидентных $y$ (если таких ребер нет, то положим $\left.p_{y}=0\right)$. 
Теперь мы повторим рассуждения, приведенные выше для случая подвижной вершины $x$, заменив в них $\bar{\partial} H$ на $\bar{\partial} H \cup\{y\}$ и заменив сумму $p_{y}$ импульсов в вершине $y$ на $p_{y}-p$. Отметим, что сумма так переопределенных импульсов в вершинах из переопределенного множества $\bar{\partial} H$ теперь равна нулю. Кроме того, неравенства (1) остаются справедливыми, так как значение поля деформации в неподвижной вершине $y$ равно нулю. Таким образом, мы находимся в тех же предположениях, что и в случае подвижной вершины $x$. Доказательство закончено.

ПримеР. Пусть $\Psi_{L}-$ функционал длины на римановом многообразии $W$. Очевидно, мы находимся в условиях теоремы 3.2. Поэтому в качестве следствия получается описание локальной структуры экстремальных следов, см. [3] или [4].

СлЕДСТВИЕ 3.6. Сеть-след $\Upsilon$ с границей $\Upsilon$ на римановом многообразии $W$ является әкстремалью функиионала длины, если и только если выполнены следующие условия:

1) ребра сети $\Upsilon$ - геодезические;

2) угол между любыми двумя смежными ребрами больше или равен $120^{\circ}$;

3) каждая вериина степени 1 принадлежст д؟;

4) если вершина степени 2 не принадлежит $\Upsilon \Upsilon$, то угол между иниидентнымми ей ребрами равен $180^{\circ}$.

Отметим, что первое и второе условия следствия 3.6 вытекают соответственно из первого и второго условий теоремы 3.2. Третье и четвертое условия следствия 3.6 вытекают из третьего условия теоремы 3.2. Четвертое условие теоремы 3.2 для функционала длины вьполняется автоматически.

\section{СПИСОК ЦИТИРОВАННОЙ ЛИТЕРАТУРЫ}

[1] Ivanov A. O., Tuzhilin A. A. Branching Solutions of One-Dimensional Variational Problems. World Publisher Press, 2000 (to appear).

[2] Дубровин Б. А., Новиков С. П., Фоменко А. Т. Современная геометрия. М.: Наука, 1986.

[3] Иванов А. О., Тужилин А. А. Геометрия минимальных сетей и одномерная проблема Плато // УМН. 1992. Т. 47. № 2 (284). С. 53-115.

[4] Ivanov A. O., Tuzhilin A. A. Minimal Networks. The Steiner Problem and Its Generalizations. N.W., Boca Raton, Florida: CRC Press, 1994. 